\section{Double Molecules in Gases}

WE have observed double molecules, for example $\left(\mathrm{CO}_{2}\right)_{2}$, in carbon dioxide, nitrous oxide, nitrogen, oxygen, argon and xenon. It is believed that these double molecules exist in all gases at temperatures where the attractive Van der Waals interaction is of importance, and where the pressure is sufficiently high to produce a significant fraction of three-body collisions. Their existence has been predicted for many years ${ }^{1}$.

A mass spectrometer was used to examine the spectra of the various gases in which these species were observed. Gas from a reservoir at pressures up to 200 torr passed through a narrow orifice (diameter and length $\leqslant 2 \times 10^{-4}$ $\mathrm{cm}$ ) directly into the ionizing electron beam of the spectrometer. As the mean free path of the molecules in the gas was comparable to, or greater than, the orifice diameter, the flow was of molecular type, and so the double molecules could not be formed by the adiabatic cooling of the gas ${ }^{2}$. There is substantial evidence that the observed double molecules are not the products of ion-molecule or excited state reactions in the mass spectrometer. For example, it was found that the measured concentrations at constant pressure were almost independent of the variations in gas flow resulting from altering the orifice diameter. The energy of the electron beam was chosen to eliminate the possibility of apparent double-mass ions caused by charge exchange between doubly charged ions and molecules ${ }^{3}$.

For argon and xenon, the concentration of double molecules, and their variation with temperature over a range of $180^{\circ}-300^{\circ} \mathrm{K}$, agree reasonably well with the values computed by Stogxyn and Hirschfelder ${ }^{4}$ for the Lennard-Jones (6-12) interaction potential. As an example, the concentration of $\mathrm{Ar}_{\text {, in }}$ argon at $300^{\circ} \mathrm{K}$ and 100 torr was about $10^{-4}$. In the case of the polyatomic molecules, the measured concentration is much less than predicted. This is associated with an instability of the double molecules in their flight from the reservoir to the electron beam.

This obsorvation of double molecules in gases provides direct experimental evidence for the physical reality to be accorded the concept of 'clusters' in the statistical mechanical treatment of imperfect gases. These dimers account for a major fraction of the second virial coefficient in gases below their Boyle temperature, and can be regarded as the first condensation embryos which are always present in a gas, even in its unsaturated state.

We thank the Smethurst High-Light Co., who supplied us with the small orifices, and also the management of the Reactor Group, U.K. Atomic Energy Authority, for their support of this work.

\section{E. J. Robbins \\ R. E. LeCkenby}

Reactor Engineering Laboratory,

U.K. Atomic Energy Authority, Risley, Warrington.

${ }_{1}$ Jeans, J. H., The Dynamical Theory of Gases (Cambridge Univ. Press, 1904) ${ }^{2}$ Leckenby, R. E., Robbins, E. J., and Trevalion, P. A., Proc. Roy. Soc., A 280, 409 (1964).

sorton, F. J., Mass Spectroscopy in Physics Research, Nat. Bur. Stand. Circ., $522(1953)$.

${ }^{4}$ Stogryn, D. E., and Hirschfelder, J. O., J. Chem. Phys., 31, No. 6, 1531 (1959).

\section{BIOCHEMISTRY}

\section{Rapid Separation of Mucopolysaccharides by Salt-gradient Ion-exchange Paper Chromato- graphy}

EXCELLENT separation of acid mucopolysaccharides can be obtained by elution from columns of 'Dowex 1 Chloride'1 or 'DEAE Sephadex'2 with sodium chloride solutions of increasing molarity. The mucopolysaccharides are detected in the column effluents by uronic acid or hexosamine assays. Rolatively large $(5-10 \mathrm{mg})$ samples of individual mucopolysaccharides are, however, required for these methods.

Much smaller $(10-20 \mu \mathrm{g})$ samples may easily be separated on ion-exchange paper using ascendinis chromatography with a sodium chloride solution of steadily increasing molarity, and subsequent detection of the samples with alcian blue.

The apparatus is similar to that used by Marsden and Kent $^{3}$ for the separation of mucopolysaccharide-cetylpyridinium chloride complexes on paper. A Petri dish (15 cm diam.) containing $50 \mathrm{ml}$, water and a magnetic stirrer is placed in a chromatography tank $(40 \mathrm{~cm} \times$ $20 \mathrm{~cm} \times 20 \mathrm{~cm}$ ). Samples of mucopolysaccharides $(10-20 \mu \mathrm{g})$ are applied as spots to an 'origin' line drawn $2 \mathrm{~cm}$ from the lower end of a $10 \mathrm{~cm} \times 30 \mathrm{~cm}$ strip of 'Amberlite $S B$ 2' ion-exchange paper. The lower end of the paper is dipped into the Petri dish, and the magnetic stirrer is switched on. At the same time, a 4-M solution of sodium chloride in water is delivered into the Petri dish at a rate of $60 \mathrm{ml}$./ $\mathrm{h}$ by means of a micropump (Distillers Company Series II) and a fine polythene catheter which passes through the lid of the chromatography tank into the Petri dish. Ascending chromatography is allowed to proceed for $1.5 \mathrm{~h}$. The ion-exchange paper is then dried in a current of warm air, stained with a 1 per cent solution of alcian blue (G. T. Gurr) in 2 per cent acetic acid (15 min) and washed in briskly running tap water $(30 \mathrm{~min})$.

In a typical run under these conditions, in which the solvent front moved $28.3 \mathrm{~cm}$, hyaluronic acid (human aorta) migrated $18 \mathrm{~cm}$ from the origin $\left(R_{F} 0 \cdot 63\right)$, heparitin sulphate (human aorta) migrated $14 \mathrm{~cm}\left(R_{F} 0 \cdot 49\right)$, chondroitin sulphate $C$ (human aorta) migrated $9 \mathrm{~cm}\left(R_{F} 0 \cdot 32\right)$ and heparin (Evans Medical, Ltd.) migrated $7 \mathrm{~cm}\left(R_{F}\right.$ 0.24). The disadvantages of the method are that the paper is easily overloaded with mucopolysaccharide, with consequent upward tailing of the spots, and chondroitin sulphates $A, B$ and $C$ cannot be separated from each other.

The method of choice for the separation of very small quantities of mucopolysaccharides is probably electrophoresis on cellulose acetate membran $e^{4,5}$, but the method described here provides useful additional information when mucopolysaccharide samples are too small to allow conventional chemical characterization of their components.

Nuffield Department of Clinical Biochemistry, Radcliffe Infirmary, Oxford.

${ }^{1}$ Schiller, S., Slover, G. A., and Dorfman, A., J. Biol. Chem., 236, 983 (1961). ${ }^{2}$ Schmidt, M., Biochim. Biophys. Acta, 63, 346 (1962).

8 Marsden, J. C., and Kent, P. W., Nature, 194, 573 (1962).

4 Manley, G. W., and Kent, P. W., Brit. J. Exp. Path, 44, 635 (1963).

s Gore, I, Tanaka, Y., and White, H. A., Arch. Path., 78, 181 (1964).

\section{Enzymatic Synthesis of Cyclo propane Fatty Aldehydes}

FATTy aldehydes containing a cyclopropane ring have been isolated after acid methanolysis of the phospholipids obtained from Clostridium butyricum. 36 per cent by weight of the fatty aldehydes contained a cyclcpropane ring ${ }^{1,2}$. Zalkin et al. ${ }^{3}$ and Chung and Law ${ }^{4}$ have shown that cyclopropane fatty acids are formed by the addition. of a methyl group from $S$-adenosylmethionine to an unsaturated fatty acid moiety of phosphatidylethanolamine. An enzyme which catalyses this addition has been purified from extracts of $C$. butyricum ${ }^{4,5}$. The vinyl ether-linked, aldehydogenic chains in $C$. butyricum plasmalogens can be derived from exogenous fatty acids and fatty acid precursors, presumably by way of tho normal pathways for fatty acid synthesis ${ }^{2}$.

The formation of cyclopropane aldehydogenic moieties could occur by at least two pathways: (1) conversion of a cyclopropane fatty acid moiety to an aldehydogenic moiety by reduction of either the free acid to an aldehyde 